\title{
Protective Role of Herbal Drugs in Diabetic Neuropathy: An Updated Review
}

\section{ESHITA SHARMA ${ }^{1}$, TAPAN BEHL ${ }^{1 *}$ MONIKA SACHDEVA ${ }^{2}$, RASHITA MAKKAR $^{1}$ AND SANDEEP ARORA ${ }^{1}$}

${ }^{1}$ Chitkara College of Pharmacy, Chitkara University, Rajpura, Punjab-140401

${ }^{2}$ Senior Lecturer, Fatima College of Health Sciences, AL Ain, United Arab Emirates

*Email: tapan.behl@chitkara.edu.in

Received: December 20, 2017 I Revised: February 18, 2018 I Accepted: March 11, 2018

Published online: May 02, 2018

The Author(s) 2018. This article is published with open access at www.chitkara.edu.in/publications

Abstract: Medicinal plants play a beneficial role in health care and are commonly used in preventing and testing diseases and specific ailments. The advantage associated with herbals plants are numerous and cannot be ignored as they have less adherence issues and are accepted widely by the population due to greater belief in Ayurveda since ancient times. Neuropathic pain has immersed as a serious threat to patient that occurs by damaging the blood vessels leading to morbidity and mortality. The present review paper aims in providing an account of various herbal plants that could be employed in treatment of neuropathic pain.

Keywords: Diabetic neuropathy, Pyncnogenol, Salvia miltiorrhiza, Anisodus tanguticus, Glycine max, Ginkgo biloba

\section{INTRODUCTION}

Diabetes is a fatal disorder of protein, fat and carbohydrate metabolism that is characterised by increase in fasting and post prandial blood sugar level. On a global level there has been an estimate of increase in diabetes from $4 \%$ to $5.5 \%$ by the year 2025 (Cogan et al., 1961).Various studies conducted across India in the last few decades highlighted that it is not only prevailing but also increasing on a rapid rate in the urban population. It is estimated that approximately 33 million adults are affected with diabetes in India and this number is likely to increase up to 57 millionby the year 2025. It is a multifactorial diseases leading further to many complications, therefore demands a multiple therapeutic approach (Cogan et al., 1961)
Journal of Pharmaceutical Technology, Research and Management

Vol-6, No-1, May 2018 pp. 21-30 
Sharma, E

Behl, T

Sachdeva, M

Makkar, R

Arora, S

Diabetic neuropathy refers to nerve damage that occurs due to overtime high glucose level in the blood. It is also termed as sugar and is responsible for damaging nerves. The prophecy of occurrence of this disease can be made by the duration of diabetes itself. There are various symptoms faced by the patients such as numbness, pain in feet or in the lower part of legs sometimes leading to loss of sensation in the feet further which may develop to ulcers or lesions if gone unnoticed. Depending upon the part of body affected diabetic neuropathy is of various types such as peripheral neuropathy, autonomic neuropathy, focal neuropathy and proximal neuropathy (Ramachandran et al., 2002). Peripheral neuropathy is so common that it has affected almost onehalf population with diabetes. It affects legs and sometimes hands. Autonomic neuropathy damages nerves controlling our internal organs, leading to problems related to heart, digestive system, sweat gland and eyes. This may further lead to hypoglycaemia if left unnoticed. Focal neuropathies typically affect mainly single nerves of hand, head, torso or leg and may lead to carpal tunnel syndrome (Dateo et al.,1973). Proximal Neuropathy damages nerves of hips, thigh or buttocks affecting mainly one side one the body which may further spread to the other side (Figure 1).

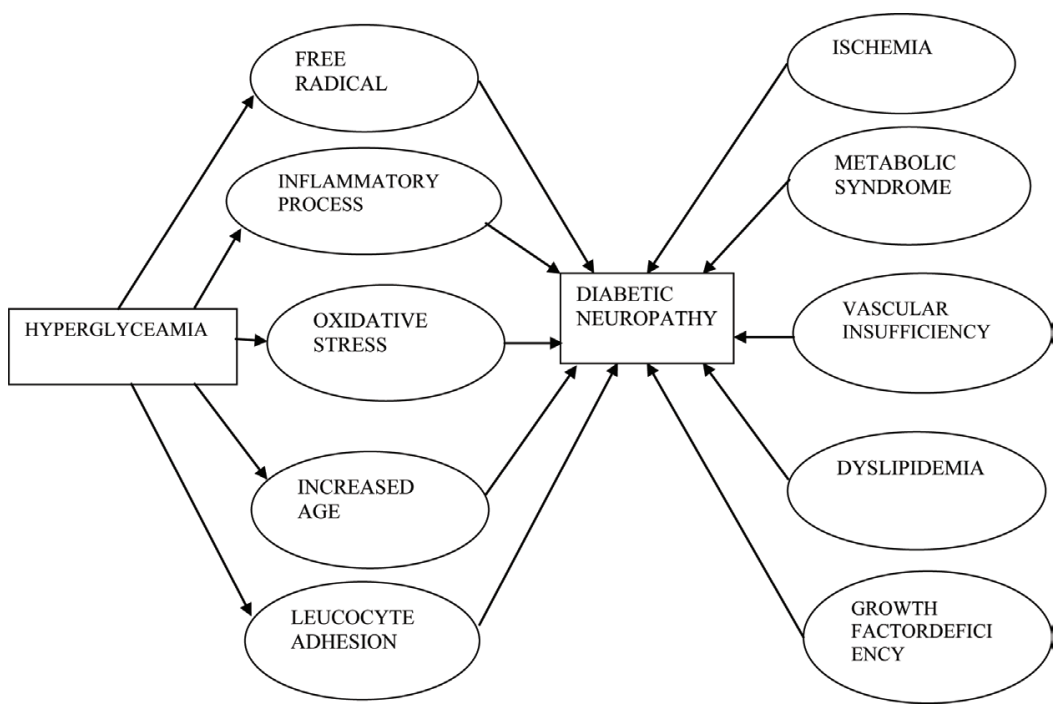

Figure 1: Mechanisms involved in development of Diabetic Neuropathy

\section{ROLE OF HERBAL PLANTS IN MEDICINES}

Plants are always termed as the best source for medicines. Plants provided us with all the basic necessities to survive such as medicines, food and fresh 
air to breathe. The active chemical constituents obtained from plants can be used in various forms. These active chemicals can directly be used in for pharmacological activity to fight against diseases. Their derivatives can also be extracted for the same purpose. In total approximately 400 different types of plants are known to be used to fight against diabetes (Tredici, 2000). Since in the last few decades herbal medicines have gained a lot of importance, therefore few of the herbal plants are listed below with their use in pharmacology to fight against diabetes. Many conventional drugs have been derived from prototypic molecules that are present in medicinal plants. They provide us with a pool of alternative medicine system or supplement to conventional therapies of various disorders. Till date, over 400 traditional plant treatments have been reported for plant diabetes, though only a small number out of these have received medical and scientific evaluation to assess their efficacy (Deng et al., 2005). The hypoglycaemic effect of some of the herbal extracts has been confirmed in human and animal bodies suffering from diabetes. The World Health Organisation Committee on diabetes has recommended that traditional medicinal herbs be furthermore investigated. The herbals plants mentioned in the present review have shown promising results in the attenuation of diabetic neuropathy by extensive research done on them over the years.

\subsection{Pinus pinaster}

It is the family member of Pinaceae most commonly referred to as Maritime Pine Tree, native to Mediterranean region of Europe (Feldman et al., 1970). A derivative is extracted from the outer bark of the plant and is called Pycnogenol. It is basically a group of compound containing proanthocyanidins and some other constituents such as catechin, taxifolin and few acid derivatives mainly of phenol. Also few trace metals are present in it such as calcium, iron, potassium etc.

- It increases the membrane integrity of the capillaries and is able to do so because of the procyanidins present in it coming under non-hydrolysable tannins class. It has a property of binding to proteins of cellular membrane. Hence stabilizing the walls of blood capillaries and further preventing the same from damaging (Figure 2).

- It has a capability of being a potent antioxidant due to free radical scavenging activity thus resulting in lowering amount of Reactive oxygen Species (ROS) inside our body which further leads to glycation products activation due to glycolytic metabolites accumulation.

- It also poses a property of radical scavenging of pycnogenol which is further responsible for its anti-inflammatory property. By using inflammatory mediators it reduces the degeneration of capillaries.
Protective Role of Herbal Drugs in Diabetic Neuropathy: An Updated Review

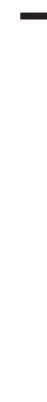


Sharma, E

Behl, T

Sachdeva, M

Makkar, R

Arora, S
- It has also has few other compounds which decrease the amount of advanced glycationend-products (AGEs). These compounds are responsible for neuropathic pain among patients (Feldman et al., 1970).

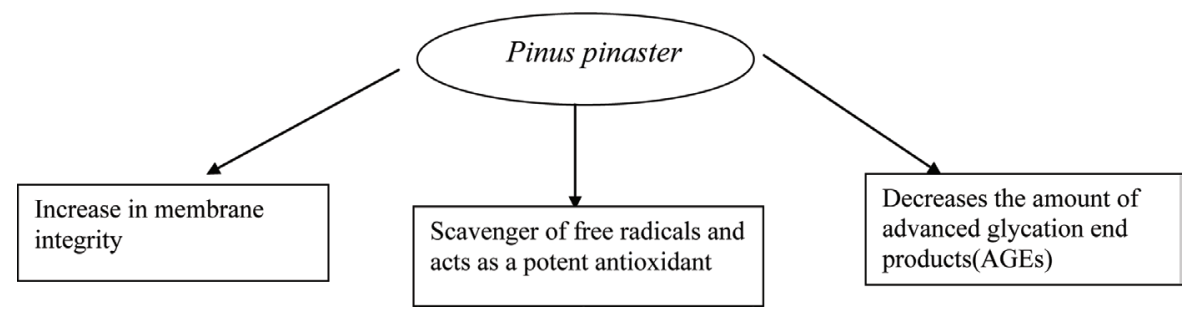

Figure 2: Mechanism of action of Pinuspinasterin prevention of diabetic neuropathy

\subsection{Salvia miltiorrhiza}

Salvia miltiorrhiza commonly known as Danshen or Chinese sage belongs to Lamiaceae family and is a perennial plant native to Japan and china therefore used in Chinese medicines. Polar phenolic compounds of lipophilic nature are major constituent of this plant which are known as tanshinones which includes tanshinone I, tanshinone II A. Phenolic acids like caffeic acid are obtained from polar phenolic compound whose derivatives are salvianolic acid A, danshensu, rosemaeinic acid, salvianolic acid B and prolithospermic acid (Packer et al.,1999), (Pershaud et al.,1999). The anticoagulant and antioxidant properties is due to the polar compounds and the antibacterial and antineoplastic property is due to presence of lipophilic compounds.

- It has the ability to control leakage of blood from small blood capillaries that supply the blood to various parts of the body.

- Hemorrhages and magnitude of micro aneurysms decreased as compared to with that of its earlier conditions, thus the pills made out of these plant were further used in neuropathic pain treatment (Figure 3).

- Ischaemia prevalent in blood capillaries carrying blood was seen to improve by the use of danshen pills (Wang et al., 2007). The use of these pills further gave positive results in treatment of diabetes.

\subsection{Anisodus tanguticus}

Anisodus tanguticus commonly known as ZangQie is native to China and is also called as Scopolia tanguticus which is a perennial flowering plant that belongs to Solanaceae family (Zhao et al., 2011). It contains tropane 


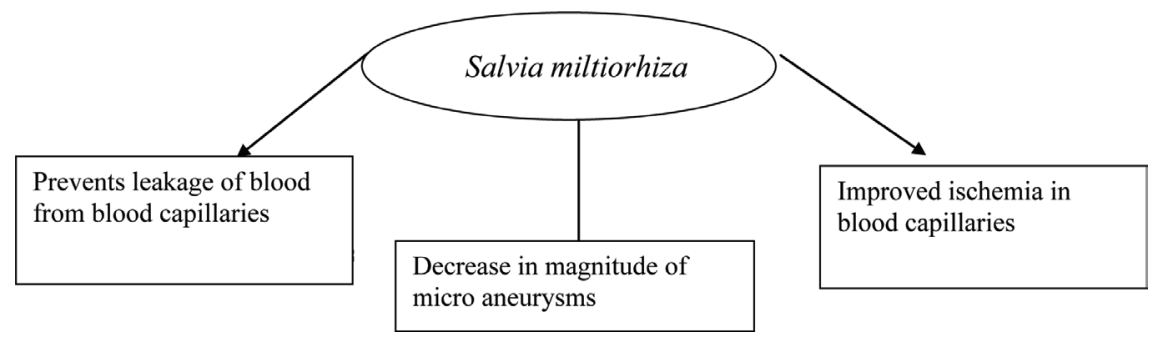

Protective Role of Herbal Drugs in Diabetic Neuropathy: An Updated Review

Figure 3: Mechanism of action of Salvia miltiorhiza in prevention of diabetic neuropathy

alkaloids like anisodine, hyoscyamine, anisodamine, scopolamine, tropine and apart from all these constituents it also contains a non-tropane alkaloid cusgohygrine. Among these anisodamine is the most active compound of this plant whose derivatives show various anti-cholinergic and pharmacological activities.

- The fibrinolytic activity of anisodomine present in the plant helps in reversal of endotoxin induced vascular leakage that improves blood supply to the various organs resulting in inhibiting the inflammation in capillaries.

- Anisodomine is also known to have self protective action that inhibits inflammation action which prevents haemorrhages, that commonly occurs in patients suffering from neuropathic pain (Tapsell et al.,2006).

- Anisodamine blocks muscarinic receptor by acting as a muscarinic receptor antagonist which leads to cholinergic anti-inflammatory pathway activation, contributing a modulatory role in neuropathic pain (Figure 4).

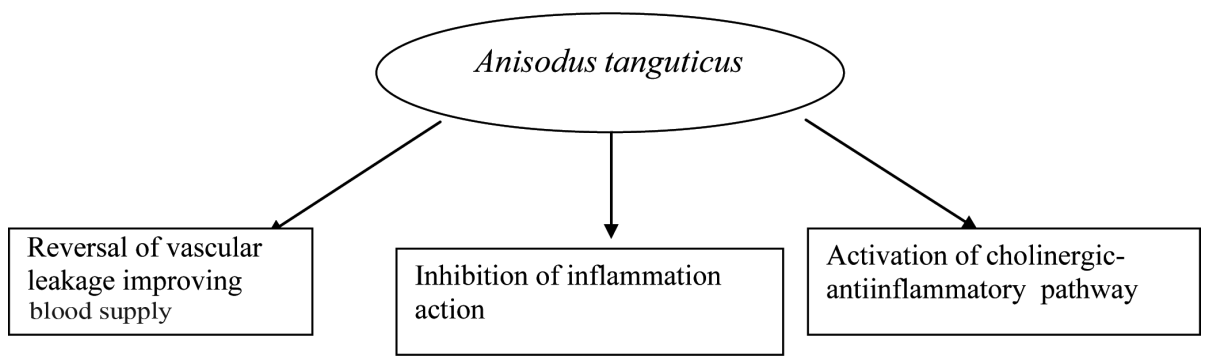

Figure 4: Mechanism of action of Anisodus tanguticus in prevention of diabetic neuropathy 
Sharma, E

Behl, T

Sachdeva, M

Makkar, R

Arora, S

\subsection{Glycine max}

Glycine max, commonly known as Soyabean belongs to Fabaceae family (Stepp et al., 2001). It helps in decreasing the diabetic neuropathy progression by preventing morphological destruction of kidney which is associated with diabetes. It performs the function of converting fatty acids intodocosahexaenoic acid which benefits in various inflammatory models and renal decreases by increasing production of complex lipids (Nguyen et al., 2012). It also decreases excretion of urinary albumin and cholesterol in subjects with neuropathic syndrome. A diet of soyabean improves the level of insulin and serum glucose thus preventing morphological kidney disruption and weight loss (Jan et al., 2012). Insoluble carbohydrates constitute 15\% of soyabean while $30 \%$ of the soluble variety is fibre hence their digestion is sloe with a low glycaemic index.

- Soybean protein has role in diabetes due to presence of glycine and argentine in it that tends to reduce the insulin modulated effects. It shows effects on hypertension, hypercholesterolemia, obesity and atherosclerosis which are common problems in patients suffering from diabetes neuropathy.

- Substituting the animal protein for soybean may also decrease proteinurea, renal hyperfiltration, and renal load thus reducing the risk of renal diseases in diabetes patients (Figure 5).

- The antiangiogenic effects of isoflavones can be related in treatment of this disorder, though soybean is associated with benefit for patients with gallstones. The mechanism can be associated to blood cholesterol lowering effects of soybean protein containing isoflavones.

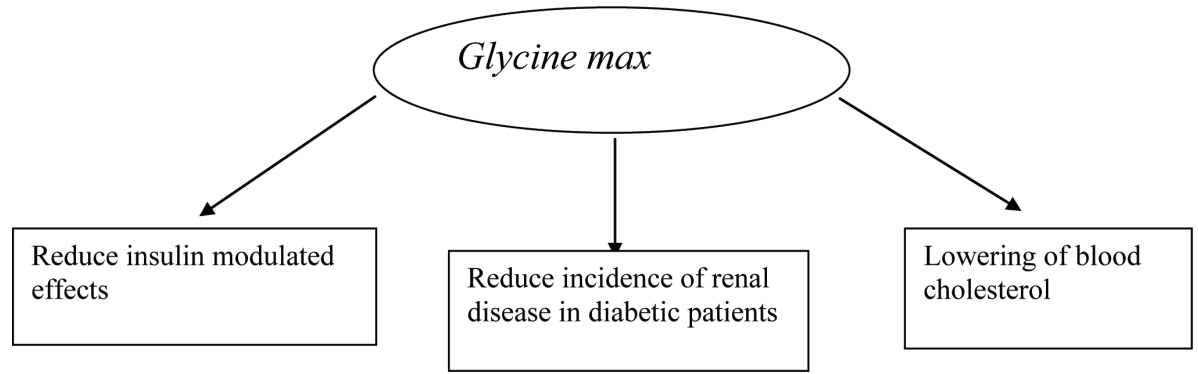

Figure 5: Mechanism of action of Glycine max in prevention of diabetic neuropathy 


\subsection{Ginkgo biloba}

It is commonly called Maiden hair Tree, belongs to Ginkgoaceae family is native to china. It is the oldest tree species prevailing on earth. Its chemical constituents are Ginkgolides, Bilobalide, flavonoids and various other compounds such as bilobetin, ginkgolic acid, ginkgetin, carotenoids, proantcyanidins, arcorbic acid, tannins, sterols, vanillic acid and fatty acids. $\mathrm{A}, \mathrm{B}, \mathrm{C}, \mathrm{J}$ and $\mathrm{M}$ are the commonly found ginkgolides out of which $\mathrm{B}$ is the most active ginkgolide that posses ginkgotoxin which is a toxic substance. This substance is known toshow anti-vitamin B6 activity (ManjulaSri and Rao et al., 2010). This particular property results in loss of consciousness and formation of convulsion.

- It reduces adhesion of leukocytes and increases the blood circulation by acting as a blood thinner (N.G. Congdon et al., 2003). Thus helping in preventing other inflammatory actions as well (Figure 6).

- It has the property of inhibiting the platelet activation because of which it plays an important role to prevent the inflammatory responses in the body of subject (Tang et al., 2002).

- The flavonoids of Gingko biloba posses anti oxidant property that helps in scavenging the reactive oxygen species in body and relieving it from oxidative stress. These actions helps in reducing hypertension and vasorelaxation in the capillaries of the patient (Grisan et al., 2006).

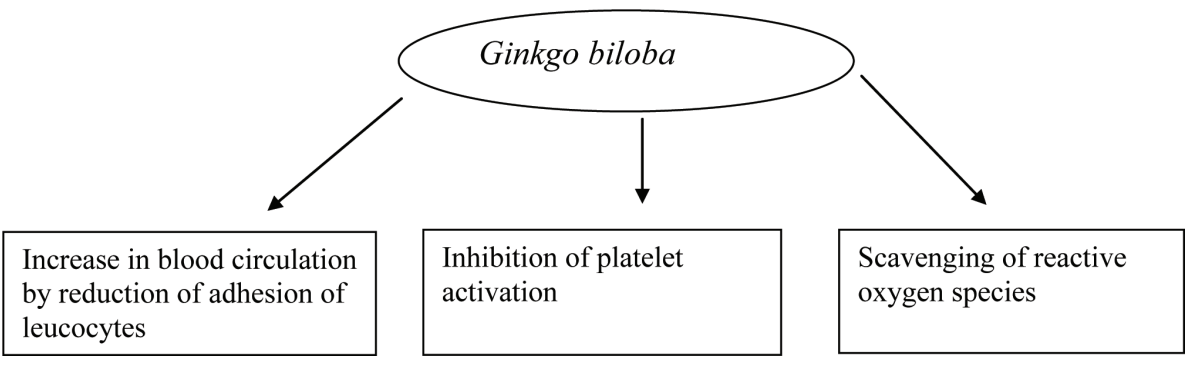

Figure 6: Mechanism of action of Ginkgo biloba in prevention of diabetic neuropathy

\section{CONCLUSION}

In recent years, there has been a tremendous growth in field of herbal medicine and these drugs are becoming popular among both developing and developed countries. This is mainly due to their lesser side effects and
Protective Role of Herbal Drugs in Diabetic Neuropathy: An Updated Review 
Sharma, E

Behl, T

Sachdeva, M

Makkar, R

Arora, $\mathrm{S}$ natural origin. Since diabetic neuropathy is a serious complication, there is an urgent need to find an effective treatment that could completely eradicate diabetic neuropathy (Awadi et al., 1987). The herbal drugs described in the study provides an overview of the alternative options which are available, that could act as a substitute to any of the modern medicine and result in management of the disorder. Thus there is a need to discover more resources that could improve the conditions of present scenario. Major hindrance in amalgamation of herbal medicine in modern medical practices is lack of scientific and clinical data proving to their safety and efficacy. There arises a need for conducting clinical research in developmentof simple bioassays for toxicological and pharmacological evaluation, biological standardization, and development of various animal models for safety as well as toxicity evaluation. It is necessary to establish the active components from various plant extracts.

There have been made a use of different plants in various formulations for treatment of diabetes and its existing complications. One of the major problem being faced with herbal formulations is that the active ingredients present in them are not well defined (Davis and Maro, 1989). There lies an importance to know the presence of active components and their existing molecular interactions. This helps in analysing the therapeutic efficacy of the product and also aids in standardisation of the product. Various efforts are being made in order to investigate the mechanism involved in action of plants by making use of model systems. Thus, in a nutshell the use of herbal drugs as a therapeutic agent in the treatment of various morbidities will provide a new paradigm to the healthcare society (Table 7).

Table 7: Herbal Drugs and their other therapeutic actions.

\begin{tabular}{cc}
\hline HERBAL DRUG & OTHER THERAPEUTIC ACTIONS \\
\hline Pinus pinaster & Osteoarthritis \\
& Migraine \\
& Male infertility \\
Glaucoma & Erectile dysfunction \\
& Edema \\
\hline Salvia miltiorrhiza & Cancer \\
& Ischemic diseases \\
& Hypertension \\
& Depression \\
& Arrhythmia \\
& Fertility \\
\hline
\end{tabular}




\begin{tabular}{cc}
\hline Anisodus tanguticus & Eclampsia \\
& Circulatory shock \\
Epidermal meningitis & Rheumatoid arthritis \\
& Glomerulonephritis \\
& Pulmonary edema \\
\hline Glycine max & Osteoporosis \\
& Anticancer \\
& Antihyperlipidemic \\
& Stomachic \\
Ginkgo biloba & Diaphoretic \\
\hline \multirow{2}{*}{ Ginxiety } \\
& Alaucoma \\
& Dementia \\
& Alzheimers \\
& Cardiomyopathy \\
\hline
\end{tabular}

Protective Role of Herbal Drugs in Diabetic Neuropathy: An Updated Review

\section{REFERENCES}

[1] Al-Awadi F.M., Gumaa K.A (1987). Studies on the activity of individual plants of an antidiabetic plant mixture. Acta Diabetologica, 24, 37-41.

https://doi.org/10.1007/BF02732051

[2] Cogan, D.G., Toussain, D., Kuwabara T.(1961). Retinal vascular patterns: IV. Diabetic retinopathy. Arch.Ophthalmol., 66, 366-378.

\section{https://doi.org/10.1001/archopht.1961.00960010368014}

[3] Congdon N.G. (2003). Important causes of visual impairment in the world today. JAMA: The Journal of the American Medical Association, 290, 2057-2060. https://doi.org/10.1001/jama.290.15.2057

[4] Dateo, G.P., Long, L. (1973). Gymnemic acid, the antisaccharine principle of Gymnema sylvestre. Studies on the isolation and heterogeneity of gymnemic acid A1. J.Agric.Food Chem., 21, 899-903. https://doi.org/10.1021/jf60189a030

[5] Davis R.H., Maro N.P. (1989). Aloe vera and gibberellins, Anti-inflammatory activity in diabetes. J. Am. Pediat. Med. Assoc., 79, 24-26.

https://doi.org/10.7547/87507315-79-1-24

[6] Del Tredici, P. (2000). Ginkgo biloba. Gordon and Breach Publishing Group, Inc., New York, 274.

[7] Deng, H., Jin, M., Yuan, W., Xiang, Z., Wang J.(2005). Clinical observation treating early diabetic retinopathy with compound danshen dripping pills. J. Traditional Chin. Ophthalmol., 15, 72-74.

[8] Feldman, J.M., Lebovitz, H.E. (1970). Mechanism of epinephrine and serotonin inhibition of insulin release in the golden hamster in vitro. Diabetes, 19, 480-486. https://doi.org/10.2337/diab.19.7.475 
Sharma, E

Behl, T

Sachdeva, M

Makkar, R

Arora, S
[9] Grisan E. (2006). Model-based illumination correction in retinal images in Biomedical Imaging: Nano to Macro. 3rd IEEE International Symposium, 984-987

[10] Jan J. Retinal image analysis aimed at blood vessel tree segmentation and early detection of neural-layer deterioration (2012).Computerized Medical Imaging and Graphics, 36, 431-441. https://doi.org/10.1016/j.compmedimag.2012.04.006

[11] Manjula Sri R., Rao D.K. (2010). Review of image processing and tele ophthalmology research projects world over. Technology Spectrum Journal, 4, 42-48.

[12] Nguyen U.T., Bhuiyan L., Park L.A. F. et al. (2012). An effective retinal blood vessel segmentation method using multi scale line detection. Pattern recognition, 46(3), 703-715. https://doi.org/10.1016/j.patcog.2012.08.009

[13] Packer, L., Rimbach G., Virgili F. (1999). Antioxidant activity and biologic properties of a procyanidin-rich extract from pine (Pinus maritima) bark, pycnogenol. Free Radical Biol. Med., 27, 704-724.

https://doi.org/10.1016/S0891-5849(99)00090-8

[14] Persaud, S.J., Al-Majed, H., Raman A., Jones, P.M. (1999). Gymnema sylvestre stimulates insulin release in vitro by increased membrane permeability. J. Ethnopharmacol., 163, 207-212.

[15] Ramachandran A., Snehalatha C., Viswanathan V (2002). Burden of type 2 diabetes and its complications- the Indian scenario. Curr. Sci., 83, 1471-1476.

[16] Stepp, J.R., Moerman, D.E. (2001). The importance of weeds in ethnopharmacology. Journal of Ethnopharmacology, 75(1), 19-23. https://doi.org/10.1016/S0378-8741(00)00385-8

[17] Tapsell, L. C., Hemphill, I., Cobiac, L. et al. (2006). Health benefits of herbs and spices: the past, the present, the future. Med. J. Aust., 185(4), 4-24.

[18] Teng T. (2002). Progress towards automated diabetic ocular screening: a review of image analysis and intelligent systems for diabetic retinopathy (2002). Medical and Biological Engineering and Computing, 40, 2-13.

https://doi.org/10.1007/BF02347689

[19] Wang, X., Morris-Natschke S.L., Lee, K.H. (2007). New developments in the chemistry and biology of the bioactive constituents of Tanshen. Med. Res. Rev., 27, 133-148. https://doi.org/10.1002/chin.200719248

[20] Zhao, T., Li, D.J. Liu C., Su D.F., Shen, F.M. (2011). Beneficial effects of anisodamine in shock involved cholinergic anti-inflammatory pathway. Frontiers Pharmacol., 2. https://doi.org/10.3389/fphar.2011.00023 